\title{
Construction of Credible Ubiquitous P2P Content Exchange Communities
}

\author{
Yuki Yokohata, Hiroshi Sunaga, and Hiroyuki Nakamura \\ NTT Network Service Systems Laboratories, NTT Corporation, \\ 3-9-11 Midori-cho, Musashino-shi, \\ Tokyo 180-8585, Japan \\ yokohata.yuki@lab.ntt.co.jp
}

\begin{abstract}
This paper describes methods for suppressing illegal behavior in P2P networks to construct ubiquitous $\mathrm{P} 2 \mathrm{P}$ content exchange communities. Although rigid digital rights management has been established elsewhere, it requires rather a large processing load and time, and it is mainly effective at preventing illegal behavior of end users. Here, we propose a more efficient method for processing content distribution. It aims to control content exchange so that illegal activities can be reduced to a sufficiently low level. By observing content exchange interactions in rendezvous points, it detects illegal activities and identifies which peer performed them. Simulation results show the effectiveness of the proposal.
\end{abstract}

\section{Introduction}

We are now at the dawn of the era of a ubiquitous communication society, where our daily lives are becoming more and more dependent on various types of telecommunications. In this society, broadband IP networks will play a key role in supporting the social infrastructure as well as mobile communication networks. One of the most promising applications is content exchange, by which various types of content, created by professionals, semi-professionals, or amateurs, will be traded anywhere.

Moreover, a large majority of Internet users have experience in using or are even now using peer-to-peer (P2P)-based file exchange. Most P2P-based applications are illegal, and $60 \%$ of users feel anxious about illegal activities such as fraudulent electronic transactions, according to a report by the Ministry of Internal Affairs, and Communications (MIC), Japan [1]. However, P2P is technically promising and there must be potential users for legitimate content exchange services. In other words, if a legal community for content exchange is provided by a trusted service provider, or created only by trusted members, then legitimate content exchange markets will flourish.

As we can see from this background, various methods for achieving reliable operation of the community are necessary. Although there are various types of illegal behavior by members in a ubiquitous or P2P community, this paper focuses on how to obtain information about the status of content exchange and illegal behavior in the community, which will lead to stable and reliable operation and accelerated content-trade activities.

The rest of this paper is organized as follows. Section 2 clarifies problems and requirements for ubiquitous content exchange. In Section 3, we propose a new 
framework for identifying the status of the community, in particular illegal behavior by members. Section 4 presents simulation results and analyses. Finally, in Section 5, we present conclusions and show future directions.

\section{Problem Analysis and Requirements}

\subsection{Objectives of Ubiquitous Content Exchange Community}

A ubiquitous content exchange community is made up of users, content creators, and operators. The creators may want to sell contents that they created either originally or by modifying the content of another creator. They may sometimes deliver content for free to demonstrate their talent. When other people use this content, they may want to clarify their own rights and conditions of use. Content may be original or modified music, pictures, or movies for mobile terminals, ordinary PCs, or home information appliances. Even content created by non-professionals has potential for trade. Users may want to obtain desired content, or value-added content. Of course, both users and creators want to communicate with those who can be trusted. The community operator mediates between them by providing a safe trading environment, which leads to a new business model. It is essential to observe the status of content exchange, to trace and exclude illegal members, and to manage charging or settlement in the community.

\subsection{Problems to be Solved}

We assume that the content exchange community takes a P2P-form and is connected to authorities for the identification of users and content. Figure 1 shows a typical sequence for content exchange in a $\mathrm{P} 2 \mathrm{P}$ community. In this processing, there are several types of illegal activities that can occur, as explained below.

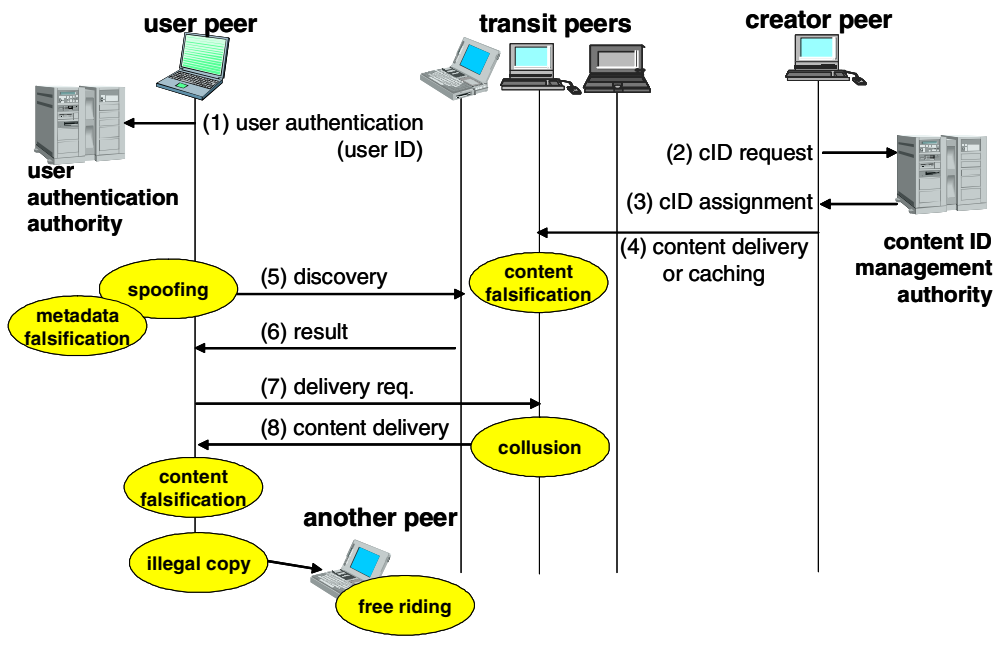

Fig. 1. This shows content exchange sequence and problems 
(a) Content falsification: content transferred through transit peers might be falsified and falsified content might be cached at a transit peer.

(b) Spoofing: some peer might spoof its address (i.e., its identity) and gain access to content illegally.

(c) Metadata falsification: during discovery processing, metadata might be falsified to make fake content that imitates copy-righted one.

(d) Collusion: a peer might send paid-for content to a partner peer that is not eligible to receive it.

(e) Illegal copying and free riding: a peer might deliver illegally obtained content to other peers, that is, free-riders.

\subsection{Existing Countermeasures}

There are three approaches to deal with these problems, i.e., prevention of illegal behavior, suppression of illegality, and permission to use content with some conditions. The most appropriate method depends on the value of content, the application type, the content holder's policy, or the operator's policy.

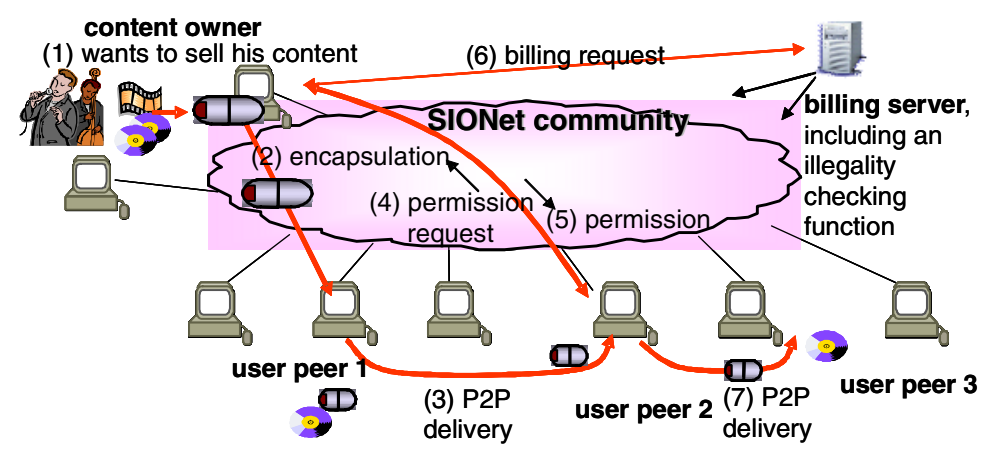

Fig. 2. This shows the P2P DRM model

\section{Prevention of illegal behavior}

This is a rigid type of countermeasure that does not allow content to be viewed without permission. Figure 2 is a rigid approach to protect the copyright of content [2]. A content owner first encapsulates his content with necessary information such as the number of times that users may view it. This capsule is viewed only through a proprietary browser and cannot be opened without his permission. Then, the capsule is delivered to a requesting peer in the $\mathrm{P} 2 \mathrm{P}$ mode, and this peer asks the owner for permission. If the user intends to pay for the content, the owner returns the permission. Upon receipt of the permission, the user can view the content. Until the specified time is reached, the content can be relayed to another peer.

This method is firstly applicable to countering illegal copying and free riding. Although it is also effective against content falsification, illegally obtained content can be exchanged by this rigid capsule. Spoofing and collusion are meaningless because the viewer must pay to view the content. It is not considered to be effective against 
metadata falsification. Note that encapsulating content takes a long time and involves a heavy processing load.

\section{Suppression of illegality}

This method aims at managing or controlling content exchange so that illegal activities can be reduced to a sufficiently low level. In this paper, we mainly deal with this approach by using some mechanism implemented in content ID forum [3] from the P2P viewpoint.

\section{Permission to use content with some conditions}

This method, famous as 'Creative Commons' [4], allows users to freely view or modify content with some conditions. Eleven sets of conditions (rules) are defined according to the combination of licensing elements. Its aim is to use the flexibility of copyright law to help an active usage or secondary creation of various contents.

This approach has been implemented, for example, in the Digital Commons system [5][6].

\section{Modeling the Suppression of Illegality}

\subsection{Identification of Community Elements}

Our basic proposal is to trace content exchange interactions through the cooperation of agents residing in the community. To identify content, each of them is assigned a unique and secure identification, i.e., content ID (cID). This ID is authenticated by an authority. Also, each peer in the community is given a user ID by a user authentication authority. The authentication processing is guaranteed through the use of public key mechanisms. By using a metadata-matching-based discovery capability, as for example, $\mathrm{KaZaA}[7]$ does, peers or content can be found based on properties given in the form of metadata, such as MPEG-7[8].

\subsection{Operational Policies}

We decided upon the following operational policies to meet the objectives.

- The basic operation is to log cIDs and metadata exchanged in the community. Logged information can be used to judge whether or not the license, price, or usage conditions of content have been falsified.

- The use of a user ID prevents spoofing. The cID is linked with the hash value of the content to be registered to guarantee its uniqueness. Because the same cID is given to copies when the content is duplicated, illegal content is linked with the same cID.

- Metadata used in this community contains information about conditions for secondary use of the content (XML description) because this community aims to activate content creation by feeding back incentives to the creators.

- There are two methods for assigning an identifier to content: 1) combining header descriptions and digital signature and 2) inserting an electronic watermark. Although the latter has better performance against illegal actions, the operational cost is higher. This paper treats the former method. 
Things for the creator who wants to protect his content to do are

1. getting the cID for content from the authority

2. calculating the hash value of the content

3. attaching the digital signature of cID, hash value and metadata to content

4. attaching metadata and cID to the header for searching

\subsection{Proposed Processing Mechanism}

The basic framework discussed thus far is to trace interactions of content exchange to detect peers performing illegal actions. Metadata, cID, hash vale that are attached during content delivery is logged somewhere in the community. If originating and destination peers log these, falsification is rather easy, which leads to incorrect logging or sabotaged logging. We position rendezvous point peer (RP) [9] to perform logging on the route between these peers, as shown in Figure 3.

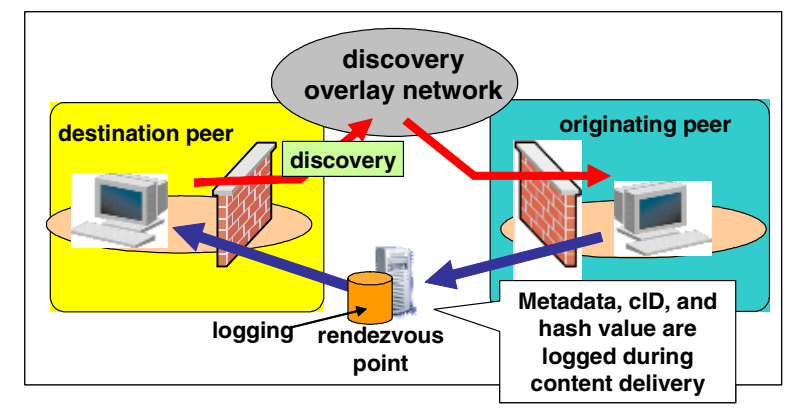

Fig. 3. This shows our proposed model of positioning rendezvous point

The obtained log data must be credible. To guarantee credibility, one peer selected by the community operator acts as the super RP peer (SRP). Its functions are to record the cID, metadata and hash value of content newly uploaded to this community and compare them with the data logged in the community. If a mismatch is detected, falsification is recognized. For example, if content is falsified, its hash value changes. A RP Peer detects the content falsification from this difference. If metadata is falsified, RP peer can detect the difference in metadata of capsules having the same content ID.

However, these SRP functions are too heavy to be performed by only one peer. Therefore, logging itself is performed in underlying RPs, managed by the SRP. RPs send logged data to the SRP at the time an ordinary peer starts content delivery. This mechanism is shown in Fig. 4.

To guarantee the credibility of the community, credible RPs must be selected. RPs are selected from ordinary peers considering the following conditions.

1. Each candidate must have a global IP address.

2. Each candidate must have higher CPU performance and a larger memory size than ordinary peers. 
3. Each candidate must leave the community less frequently than ordinary peers.

Of course, these physical conditions alone cannot prevent illegal activities. Some RPs might make an illegal linkage between cID and content, or falsify content as well as logged data.

Therefore, credible RPs are selected based on the calculated trust value. We use an equation defined in [10]. After RPs have been selected randomly from those satisfying the physical conditions, they are narrowed down to more credible RPs. The SPR compares logged data sent from RPs with original data obtained directly from the content creator. The equation of the trust value of an $\operatorname{RP}(\operatorname{Tr}(n))$ is

$$
\operatorname{Tr}(\mathrm{n})=\mathrm{a} * \operatorname{Tr}(\mathrm{n}-1)+(1-\mathrm{a}) * \operatorname{Sc} \quad(\mathrm{n}>1) .
$$

Here, ' $n$ ' means the number of interactions for content exchange and 'a' is a constant. If a match is made, then 'Sc' is increased by 1 , otherwise it is decreased by 1 .

Then, we determine a threshold value to judge whether or not falsification has occurred. If $\operatorname{Tr}(n)$ is less than threshold $h$, this means that falsification has been detected in the RP. To make this equation more effective, the following condition is required.

$$
2 \mathrm{a}-1<\mathrm{h}
$$

If $\operatorname{Tr}(\mathrm{n})$ is less than threshold $\mathrm{h}$ from the beginning, it means that an illegal RP has been selected. This RP is disqualified and another peer that has higher physical conditions is selected. Of course, at the time of selection, it is not known whether the new RP is credible. Later activities will be checked and used for judgment.

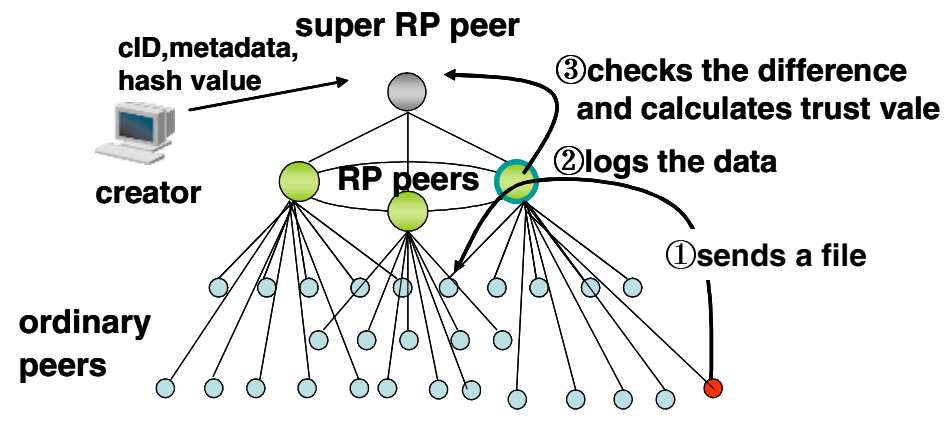

Fig. 4. This figure shows layered architecture of peers

\section{Evaluation and Analyses}

\subsection{Simulation}

We performed simulations to evaluate the proposed algorithm discussed in Section 3 . In particular, we focused on the appropriate number of RPs in a community. Then the simulator computed the number of content-exchange interactions required for a newly selected RP to detect illegal peers in the community and to augment its trust value to the 
credible level $(=1)$. This simulation identified how this value is affected by the peer configuration, i.e., the ratio of illegal actions to the total number of actions (illegal action rate) and the ratio of the number of RPs to the total number of peers in the community (RP rate). We assumed that the number of peers in the community was 1000 , the total number of interactions was 10,000 , and the parameters of equations (1) and (2) were fixed to $\mathrm{a}=0.85$ and $\mathrm{h}=0.75$ to raise the detection efficiency.

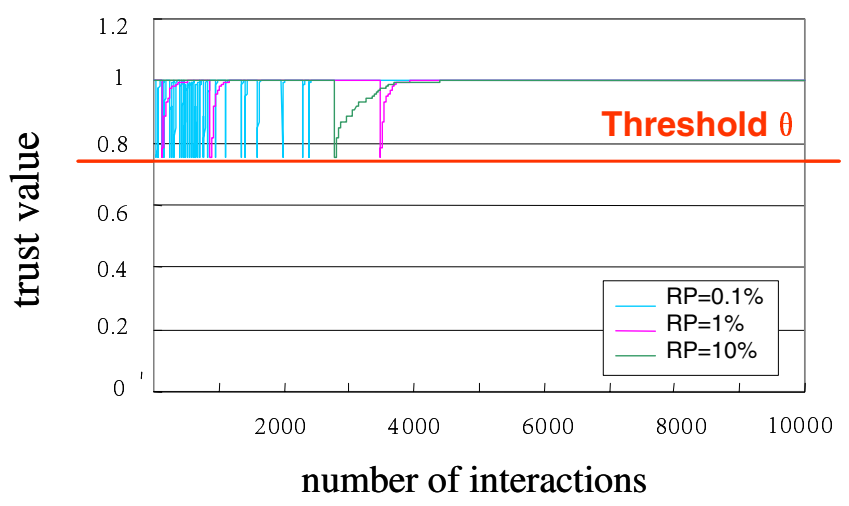

Fig. 5. This shows a figure simulated trust value for various RP peer rates

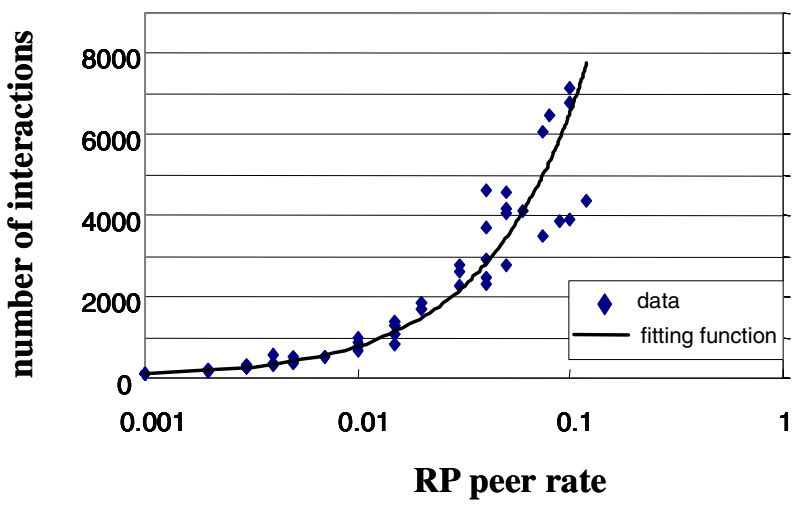

Fig. 6. Simulation result for number of interactions versus RP peer rate

Figure 5 shows how many interactions were required when the illegal peer rate was set to $3 \%$. It also compares the RP rate, which ranged from $0.1 \%$ to $10 \%$. When RPs found the illegal peers, the trust value fell according to Eq. (1).

Note that for the line for an RP rate of $0.1 \%$, the interval for detecting illegal peers becomes wider. Although it needed about 5500 interactions to detect all illegal peers when the RP rate was set to $0.1 \%$, the number of interactions required was not very different when it was under $1 \%$. We also found that the results were scattered when the $\mathrm{RP}$ rate was less than $1 \%$, but there was not much difference when it was over $1 \%$. 
Figure 6 shows how many interactions were required until the RPs' trust value could be restored when the illegal peer rate was set to $3 \%$. It took more time to restore the trust value to 1 when the RP rate was higher. This is because an RP waited for other RPs' transactions. This suggests that the number of RPs is more than required, so there are many peers that do not have sufficient tasks.

Figure 7 shows how many interactions were required when the RP rate was set $1 \%$ and the illegal peer rate ranged from $0.1 \%$ to $25 \%$. RPs could detect efficiently when there were few illegal peers, and they could do it in about 3000-4000 interactions no matter how much the number of illegal peers increased.

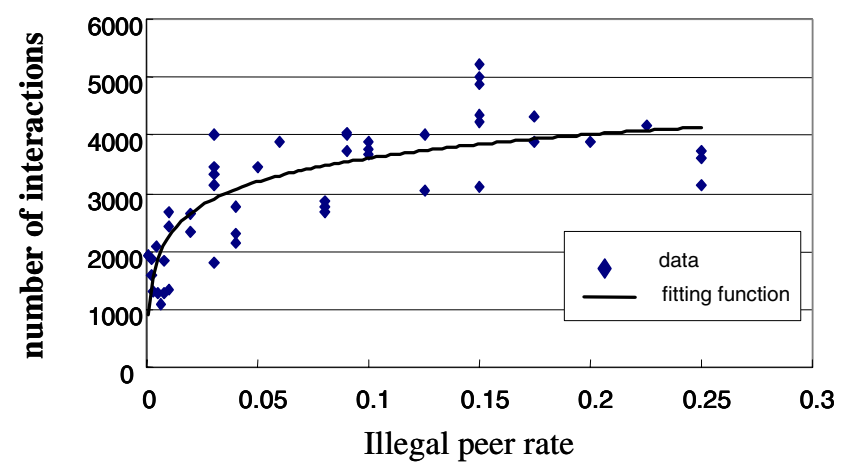

Fig. 7. Simulation result for number of interactions versus illegal peer rate

\subsection{Analyses and Consideration}

The simulation results show that the proposed algorithm could augment the trust value of each RP by evaluating behavior in the community. From our results, the most efficient number of RPs was $1 \%$ of the total number of peers. Also, RPs could detect all illegal actions among 3000-4000 interactions even if there were many illegal peers. If the rate of illegal peers in the community is less than 1\%, then 3000 interactions are needed. This suggests that $1 \%$ of RPs out of all participants is the most effective number.

Moreover, this algorithm can be applied to ordinary peers. Because the load on RPs increases as the community size grows, the ordinary peer layer should be divided into several layered groups of RPs, as shown in Figure 8. In each group, selected ordinary peers act as RPs, and if these RPs cannot perform trust evaluation, they ask the upper RPs for evaluation. This autonomous cooperation of peers has advantages of localization and network-wide load balancing as well as load reduction in the RP layer, as discussed above.

This algorithm identifies peers that are falsifying metadata or content. However, community actions after the detection depend on the community's policy. For example, illegal peers could be removed from the community or communication could be blocked at RPs or at proxies that can be controlled by the community operator. At least, warnings could be sent to the illegal peers. 


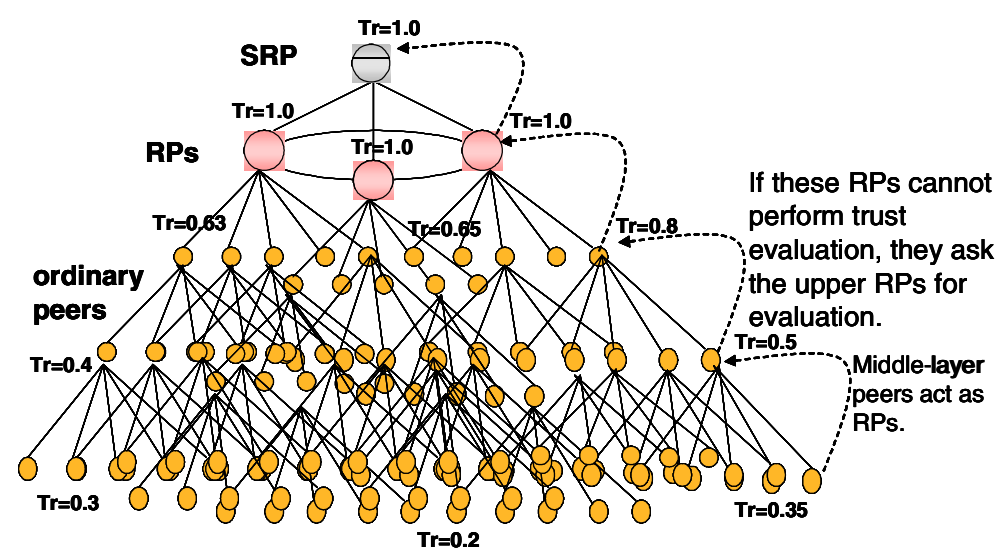

Fig. 8. This shows N-layered autonomous trust tree

\subsection{Service Applications}

The proposed illegality suppression mechanism can be applied to service operations in the future ubiquitous content exchange communities.

- Content ranking

Because cIDs are collected in RP peers, the content ranking can be created based on the cID data. The ranking data can be used as incentives to creators to create more attractive content. This service is expected to contribute to the enlargement of ubiquitous content exchange markets.

- User information management

Through data-mining of collected cID data, preferences can be identified. The results can be used to recommend content or services. Also, more specific communities can be established for people who have close preferences or properties.

\section{Conclusion}

This paper has addressed a new method for establishing a more credible ubiquitous P2P content exchange community. Although rigid digital rights management in a $\mathrm{P} 2 \mathrm{P}$ community has already been proposed, it is effective mainly for preventing illegal behavior of end users, for example, content falsification or illegal copying. Here, we proposed a more efficient method for processing content distribution. It aims to control content exchange so that illegal activities can be reduced to a sufficiently low level. By observing content exchange interactions in rendezvous points, this method can determine whether illegal activities have been performed and which peers did them. Simulation results showed the most efficient number of RPs was $1 \%$ of the total number of peers. 
We also showed some promising applications that use the data collected through this mechanism. We expect our proposals to contribute to the creation of the ubiquitous communication society.

\section{Acknowledgement}

This work was supported in part by the Research and Development Program of Ubiquitous Network Authentication and Agent (2004), the Ministry of Internal Affairs, and Communications (MIC), Japan.

\section{References}

1. MIC, Japan, “white paper on telecommunications 2005”, http://www.johotsusintoke i.soumu.go.jp/whitepaper/eng/WP2004/2004-index.html

2. T. Iwata, et al, "A DRM System Suitable for P2P Content Delivery and the Study on its Implementation," APCC 2003.

3. Content ID forum http://www.cidf.org/

4. Creative Commons http://creativecommons.org/

5. Digital Commons http://digitalcommons.jp/

6. L. Lessig, "The future of ideas," Random House, New York, 2001.

7. N. S. Good and A. Krekelberg, "Usability and privacy: A study of Kazaa P2P file-sharing," June 2002. Available at http://www.hpl.hp.com/shl/papers/kazaa/index.html

8. O. Avaro and P. Salembier, "MPEG-7 Systems: Overview," IEEE Transactions on Circuits and Systems for Video Technology, Vol. 11, No, 6, June 2001.

9. T. Oh-ishi, K. Sakai, T. Iwata, and A. Kurokawa," The Deployment of Cache Servers in P2P Networks for Improved Performance in Content-Delivery," IEEE P2P 2003.

10. Y. Wang, J. Vasseileva, "Trust and Reputation Model in Peer-to-Peer Networks," IEEE $\mathrm{P} 2 \mathrm{P} 2003$.

11. L. Xiong and L. Liu, “A Reputation-Based Trust Model for Peer-to-Peer eCommerce Communities," IEEE P2P2003.

12. R. Chen and W. Yeager, "Poblano: Distributed Trust Model for Peer-to-Peer Networks," http://www.jxta.org/docs/trust.pdf

13. B. F. Cooper, M. Bawa, N. Daswani, and H. Garcia-Molina, "Protecting the PIPE from malicious peers," Stanford Technical Report, May 2002. 